\title{
Chinese Language, Chinese Mind?
}

\author{
Christian Helmut Wenzel, Puli
}

Chinesische Gesten verstehen wir so wenig, wie Chinesische Sätze.

(We don't understand Chinese gestures any more than we do Chinese sentences.)

Wittgenstein, The Big Typescript

This article focuses on two questions. One is about the so-called Sapir-Whorf hypothesis, which says that language plays a role in how we perceive and understand the world. To put it strongly: Language determines thought. The other question is more specific. It is about the Chinese language. Is Chinese, with its grammar and its writing system, fundamentally different from Western languages such as Greek, Latin, German, Spanish or English? And does this difference account for cultural differences in perception and understanding? We all live in one world. But individually and culturally, we also live in different worlds. One easily notices this when one lives abroad. What is the role of language in this? What is its influence on perception, thought and culture? I am interested in influences that are due not just to vocabulary (which should be obvious), but, more fundamentally and systematically, to grammar and writing system. Words can easily be added to a language, one by one and step by step, and they do indeed reflect thought and culture. But grammar goes deeper. In what ways it reflects thought and culture is a more difficult but more interesting question, so it seems to me.

There has been much debate about the Sapir-Whorf hypothesis, that language determines thought. It was praised in the 1950s and 60s, argued against in the 70s and 80s, and has been returning, this time with finer tuning, since the 90s. The debate about language and thought in general is still going on, at the borderline between philosophy and linguistics.

Is it true that language somehow "determines" thought? Do we think in words and sentences, or also, and maybe more basically, in terms of feelings, pictures, associations, and memories? What exactly are words? What exactly are thoughts? Compared to thoughts, language is more on the surface. It is more accessible and less abstract. Words are visible or audible. 
They can be written down, at least so we say. But there are problems already at this level, as we shall see. Furthermore, what exactly is grammar? Does Chinese have a radically different grammar from that of Latin or English? Or is there some kind of universal grammar, so that all languages are basically the same? Does the Chinese script play a special role in this? Or could we easily change it, simplifying it by replacing it with an alphabet say, without paying a price for this, without changing the language too much and uprooting it?

\section{Psychological Differences between East and West. Stereotypes and Evidence}

There are stereotypes about East and West. It has often been said that the Chinese, Asians, or even Easterners in general, see the world as being more complex, more organic, composed of wholes and parts and interpenetrating forces (氣 Chi, 風水 Feng Shui) instead of being composed of isolated objects (atoms) that are determined by strict rules. It is said that Easterners strive for harmony more than for fairness, and that they have a greater sense of collective agency. To them the world is more dialectic (陰陽, Yin Yang), more continuous, flexible, and always changing, more cyclic and less linear in its development. They look at the whole and don't decompose and dissect so much. They have developed acupuncture and not surgery. They do not rule out contradictions from the start, but try to transcend and integrate them.

These are stereotypes, but I don't think that they are completely wrong. There are indeed differences between French, German and Chinese, or between Europe and the US. On the other hand, we must be careful with generalizations: China is not the whole of Asia: there is also Japan, for instance. Nor is Asia the whole East: there is also India. We must also be careful with drawing conclusions: Saying that one did this or that because he or she is Dutch, say, usually goes wrong. Individuals have all kinds of particular and special reasons for doing what they do. They have not only general histories and general backgrounds but also their individual ones. We cannot explain an individual person's actions so easily in terms of rough generalizations about East and West, or about being German or Chinese. Nevertheless, generalizations can make good sense, but we should learn to combine them with warning signs telling us not to jump to conclusions so easily. We must be careful when using them in arguments and in 
individual cases. To indicate that they nevertheless do make sense, and what kind of sense and how, I will give some examples.

1. Seeing a fish. To study perceptual sensitivity to background and circumstances, Taka Masuda showed a series of 20-second underwater scenes to students at Kyoto University and to students at the University of Michigan (Nisbett 2003, 89-92). In the scenes, there was a dominant fish: big, fast, or bright, and there was always some background: smaller fish, plants and bubbles. When asked what they had seen, the Japanese students mentioned more of the background than American students did. When shown additional scenes, some old, some new, some with the old background, some with a new one, and asked whether they had seen a scene before, the Japanese students recognized more scenes when not only the main fish but also the background reappeared, whereas this did not help the American students.

2. Chicken, Grass and a Cow. To compare looking at the world via relationships versus looking at it via categories, Liang-Huang Chiu (Nisbett, 140f) showed picture triplets to American and Chinese children, for instance pictures of a chicken, grass, and a cow. Does the cow belong to the grass or to the chicken? The children had to choose. Do the cow and the chicken form a group, or is it rather that the cow and the grass make up a group? American children tended to go for the first, Chinese for the second option. The first (the cow and the chicken) form a group by falling under a common heading or class (A and B are both animals). The second (the cow and the grass) form a group through interrelationship (A eats B). Classes come with rules of what counts as falling under them, but there need not be any inner connections between the members of such a class (there is no inner connection between a cow and a chicken; they do not interact). An interrelationship on the other hand is created by an inner connection. There is interaction between the cow and the grass. The cow eats the grass.

The experiment was modified by Li-Jun Ji, Zhi-Yong Zhang and Richard Nisbett, using words instead of pictures, and choosing students from the USA, Mainland China, and Taiwan (Nisbett, 141f). Again, the idea was to make the participants choose between "seeing" a common category versus seeing an inner relationship. The results were similar.

To test awareness of rules versus awareness of family resemblances, the experiment was further modified. A picture of two groups of objects together with an additional object was introduced. The additional object looked similar to all members of the two groups, but there were two kinds 
of similarity involved: On the one hand (a), there was exactly one mark by reference to which one could say that the additional object must belong to the second group. That is, one could come up with a mark, a criterion of membership for a class, so that the additional object and all objects from the second group have that very mark (and thereby satisfy that very criterion) but none of the objects from the other group did. This mark might not be so obvious. It might be minor. But it is sharp. It gives an exact criterion. On the other hand (b), one could come up with several marks (and not just one), so that the additional object and most (but not all) of the first group and very few (but not none) of the second group share them. Here we have several marks, but they are not sharp. They do not form an exact criterion (because I had to add "but not all" and "but not none"). Nevertheless, put together, they might create a greater over-all similarity between the additional object and the first group. This was the idea. Analyticity versus holism. The experiment was carried out by Ara Norenzayan, Edward E. Smith, Beom Jun Kim and Richard Nisbett (Nisbett, 141-4), and the participants were Korean, Asian American, and European American. The question was, to which group the additional object looked more similar. Sixty percent of the Koreans went for the first group, sixty-seven percent of the European Americans for the second, and the Asian Americans were in-between.

There are more experiments of this kind. They are all statistical. They do not lead to either-or rules for individuals, but they show tendencies. It is interesting and instructive to see how such experiments are set up and what they might show. Thinking through them can help us in thinking about what cultural differences could be. In particular, they show that those stereotypes I mentioned above are not completely wrong.

\section{Aristotle on Language, Mind, and the World}

In On Interpretation, Aristotle wrote that "words spoken are symbols or signs of affections or impressions of the soul; written words are the signs of words spoken. As writing, so also is speech not the same for all races of men. But mental affections themselves, of which these words are primarily signs, are the same for the whole of mankind, as are also the objects of which those affections are representations or likenesses, images, copies." (On Interpretation, 16 a) Here we see a line of representations: Written word - spoken word - soul - world. Each represents the next: Written 
words represent spoken words which represent states of the soul which, in turn, represent states of the world. We have symbols of symbols of impressions of the world: Symbols (written words) for symbols (spoken words) of impression (in the soul) of/from the outside (the world). This picture we find repeatedly and in different variations throughout history. Wittgenstein, for instance, wrote: "These concepts: proposition, language, thought, world, stand in line one behind the other, each equivalent to each.” (Die Begriffe: Satz, Sprache, Denken, Welt, stehen in einer Reihe hintereinander, jeder dem andern äquivalent. Philosophical Investigations, § 96.) We have a line of representations, symbols, or signs, reaching out to the world, and each element in this line is equivalent to the other, one standing for the next. (Wittgenstein does not subscribe to this picture. He merely presents it as a commonplace in philosophy, and he presents it in the context of his describing our striving for an ideal logical exactness in language, which he thinks is an illusion.)

In particular, that writing stands to speech as does speech to its objects, is an idea that comes out clearly in Aristotle. To quote the above passage from Aristotle again, this time in my own translation, and closer to the original: "That which is in the sound, is a symbol for the passions that are in the soul; and the written is a symbol for that which is in the sound." E$\sigma \tau 1$

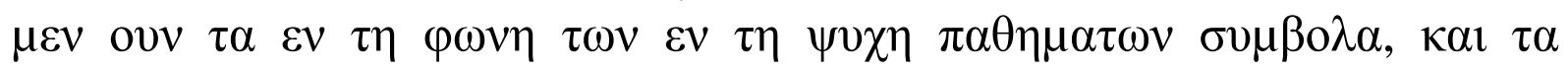
$\gamma \rho \alpha \varphi \rho \mu \varepsilon v \alpha \tau \omega v \varepsilon v \tau \eta \varphi \omega v \eta$. We first have impressions, sense impressions in our souls. Then we give names, sounds as symbols to such impressions. Finally, we learn how to write them down, in terms of symbols for those sounds. What I want to draw attention to here is that the Greek script is phonetic. There is, roughly, an isomorphism between the spoken and the written. Once you have mastered the basics of writing in Greek (or Latin, or German, or Spanish), you can write down any word that you hear, even if you hear it for the first time; and you can read and pronounce any written word you see, even if you see it for the first time. Although this does not work perfectly, and it works even less perfectly in English for instance, it roughly does work, and this is a point that is fundamentally different in Chinese. We will see in what ways it is different and what the consequences might be.

The more or less isomorphic relation between written and spoken language that we find in Western languages with phonetic (alphabetic) scripts, might have played a role in the development of Western thought about word and object in general. It might have, so I would like to suggest, en- 
couraged the view of language as being representational, a view that we find prominent in Wittgenstein's Tractatus for instance. Thinking along Aristotle's dictum, that writing stands to speech as does speech to its objects, there might have been an encouragement to view the relation between speech and object as an isomorphism of some sort as well: If there is an isomorphism between the first pair, writing and speech, why not also one between the second, speech and object? We might even speculate further. The isomorphism might encourage an atomistic view of the world: As the letters of the alphabet are like atoms, indecomposable meaningless elements, and as they represent the sounds of words that make up the spoken language, so, by analogy, the words might represent their objects, each being decomposable into elements (letters and atoms). This is all rather speculative. Nevertheless, I want to add one more speculative aspect, before returning to facts again.

A phonetic (alphabetic) script allows one to better analyze and reflect about the spoken language, because one has a way of writing down the sounds. This tool is not available in Chinese. Maybe an alphabet, due to the meaninglessness of its elements, that is, the letters, creates certain possibilities of thinking about language. All this is of course vague and speculative. But we should keep it in mind as we proceed.

\section{Chinese Language, Grammar, and Script}

First, I will give a brief and rough account of the development of the Chinese language. I do this in a way that brings out the structure that I need for my discussion. (1) The Chinese language is at its heart monosyllabic: One word, one syllable. Although Chinese has during its history increasingly combined words to make new ones, to translate foreign words, or to make words more specific, in its classical form (about 2000 years ago), and even before that, it has been monosyllabic and at heart still is so. What are the consequences of this? Including tones, there are, roughly, a little more than 1000 syllables in modern Mandarin (Putonghua). This is not enough. There are more than 1000 words. Therefore, (2) there have been, and there still are, many homonyms and homophones, words that are different in meaning but pronounced the same way. (Homonyms are also written the same, like "bank" (of a river) and "bank" (where you put your money), or in "You can drink a can of beer". Homophones are written differently, like "two" and "too", or "knew" and "new". At this point of my story, though, writing 
does not come in yet. It is only identity in pronunciation and difference in meaning that count for now.) We have this in every language. But in Chinese this is not an exception but the rule. Most words have homophones, say ten or so. If you take a sentence composed of five words, you then get $10 \cdot 10 \cdot 10 \cdot 10 \cdot 10=100.000$ possible combinations. How do we disambiguate this? How do we arrive at only one possible meaning of such a sentence? It is syntax and context that are the main ingredients here. Syntax (3) tells you that certain words preferably take certain positions. For instance, there is usually a Subject-Predicate-Object order in Chinese. (We will come back to the question, which has often been asked, whether or not Chinese has the categories of subject and predicate, or noun and verb. The answer will be yes and no, and we will say exactly in what way the answer is "yes" and exactly in what way it is "no".) Syntax helps (by disambiguating) already in conversation, through mere word order, and you do not need the script for this. Context (4) comes in different ways. There is the context in the text itself, the words that have been said before and that follow; and there is the social context, who talks to whom and on what occasion. Besides these means of disambiguation, which we find in any language, Chinese has also the script as a possibility, assigning different characters for words that are pronounced the same way. It has this option, exactly because the script is not phonetic. If it were strictly phonetic, it could only reproduce the identity in writing (homonymy). But if there is little connection between writing and sound, then there is much freedom to add and modify in writing, in order to disambiguate without impinging on the pronunciation, and this is exactly what has happened in Chinese.

In the beginning of the development of the Chinese writing, there were some signs, usually pictorial, for basic (words of) objects in the world. These signs were semantic. Then they were used (borrowed) to denote other words that happened to be pronounced the same way (creating homonyms). These signs therefore turned out to play phonetic roles. In this way, the identities in pronunciation were carried over into the writing. They were reproduced in the writing. To disambiguate the identities in writing, without touching on those in speech, additional semantic signs (S) were introduced (or already existing ones were used) and added to the phonetic ones. In this way a Chinese character usually has retained up to the present a phonetic part (P) and a semantic part (S). The semantic part is usually its so-called "radical", which serves for classification in dictionaries. It indicates a rough field of meaning, and it is this feature of a Chinese 
character that is important for what I wish to point out later.

All this sounds rather abstract. So let us look at an example. The character 衣 stands for things having to do with clothes. It covers a wide range of meaning. It serves as the semantic part (S) in the following characters: 裙 skirt, 被 blanket, 袍 coat, 裡 = 裏 lining. In the first four, we find it on the left-hand side. The fifth is a variant of the fourth: Here the clothes-radical 衣 is divided into an upper and a lower part, and the part 里 is inserted horizontally in-between. Chinese has its freedoms. 里 is the phonetic part (P). In the first four characters, the phonetic parts (P) are on the right-hand side: 君皮包里 respectively. Thus each has an SP structure, being composed of a semantic and a phonetic part.

All this is not so clean and nice as my story suggests. First, although most Chinese characters are of the semantic-phonetic (SP) type, some are concrete pictographs, abstract pictographs, or combinations of pictographs. I am focusing on the SP type here. Second, there is no exact one-to-one correspondence between fields of meanings and semantic parts (radicals). Nor is there an exact one-to-one correspondence between pronunciations and phonetic parts. Each character has its own idiosyncratic history. Each underwent changes for accidental reasons. Traces are often lost. But although these individual stories are lost, the main principles of development we know. They are the ones I have indicated. They have been applied at different stages and in different ways. Principles (of borrowing and of composing $\mathrm{S}$ and $\mathrm{P}$ parts) are not rules, and this is important here. They are not so strict. They leave more room for interpretation and change. We do not know when which principle has been applied. We will come back to this below.

With the monosyllabic structure and the introduction of characters, Chinese did not develop inflections. It did not develop suffixes to indicate case, number, person, tense, or mood. Endings would destroy the monosyllabic structure. Each word corresponds to one syllable, and in Chinese (more so than in English, for instance) it is clearly marked where a syllable begins and where it ends. There is no room for adding prefixes or suffixes. This has, so it seems to me, greatly hindered the development of that part of grammar which is called morphology. Chinese has particles (additional words that indicate the function of certain other words nearby and thus play morphological roles), but it has no inflections. It has no purely grammatical morphemes, such as "-ed" in English to indicate past tense, or “-ions” in French to indicate the first-person plural imperfect or conditional 
tense. Sometimes certain words (particles) play such roles, but there are no inflections, which would do this on a much richer scale.

Greek and Latin on the other hand are rich in this respect. Every student of Greek or Latin remembers the long tables of conjugations and declensions. These are some kind of, as I would like to call it, "systematic schemes of variation" (SSV), schemes that are to be applied to any verb or noun or adjective. Such schemes are abstract and formal. They are systematically applied to the words of the language and can be seen as a schematic meta-structure. Reflection on the presence of such schemes in Greek invites one to wonder whether this might have anything to do with the development of formal logic or axiomatic-deductive geometry as we find them in ancient Greece.

The Chinese script has no inflections, it does not have such systematic schemes of variation, but (instead, one might want to say), it has meaningful semantic parts (S) and not meaningless alphabetic letters, as in the major languages of the West. This has the consequence that the Chinese script is a much more substantial part of the language. It contributes more to the Chinese language than does the Greek script to the Greek language.

The lack of inflections implies a lack of method for indicating whether a word is a noun, a verb, or an adjective. It implies a lack of method for grammatical cross-reference within a sentence, such as that this adjective must belong to that noun, due to case and gender. In German for instance, adjective and noun can stand far apart from each other, separated by a long relative clause, without causing confusion. In that case, the two words unfold their combined meaning only during a longer stretch of time in the reader's mind, a time that is enriched by additional information, but without creating any uncertainty as to whether these two words belong together or not, and in what way they do. (Reading Thomas Mann gives a flavor of such possibilities.) There are, of course, other means of doing this: syntax, context, and particles. Nevertheless, Chinese lacks the method of inflections. It lacks this means of grammatical cross-reference within language.

This is at least one of the reasons, so it seems to me, why Chinese sentences tend to be shorter than German or Latin or Greek ones. Already in English, which does not offer as many inflections as German, it is difficult to reproduce long German sentences in translation. Kemp Smith for instance, in his ingenious translation of Kant's first Critique, has often broken up sentences into two or more separate ones. It takes much feeling to do this, i.e. to reproduce the original cross-references. Sometimes it seems 
impossible. This applies also to Chinese, maybe even more so than to English.

To indicate this lack of morphology, I shall cheat a little by using a mathematical "sentence” from a Han text on astronomy, which I take from Harbsmeier (Humboldt/Harbsmeier, 109):

二八十六三四十二。

Each of these eight signs, taken separately, stands for a number:

$\begin{array}{llllllll}2 & 8 & 10 & 6 & 3 & 4 & 10 & 2 \text {, }\end{array}$

Together, they can be read - this is only for those who know Chinese - in at least three ways, each of which is grammatically correct:
(a) $2 \quad 8 \quad 16 \quad 3 \quad 4 \quad 12$
(b) $2 \quad 80 \quad 6 \quad 3 \quad 40 \quad 2$
(c) $2 \quad 86 \quad 3 \quad 42$

Inserting multiplication and equality signs, we get various readings, among them the following:

(a) $2 \cdot 8=16,3 \cdot 4=12$

(b) $2 \cdot 80=6,3 \cdot 40=2$

(c) $2 \cdot 86=3 \cdot 42$

Only the first version is correct. We know this from mathematics. But we don't know it from Chinese grammar, and that is the point I want to bring out. The original "sentence" does not mention multiplication or equality. Words or signs for these have to be added at suitable places, and according to grammar there are at least these three options. This example might sound artificial. I do not use it to prove anything. I only use it to indicate something, namely a certain contextuality: You must understand the meaning of the words, and you must go out into the world (here the world of mathematics) to see how things are there. Only from there can you fill in the relations between the words. Let us look at some more examples. I will now give two "real" examples, namely from Laozi (71) and from Zhuangzi. Each is taken from Harbsmeier, p. 112 and p. 182, respectively. 
知不知上 know $\backslash$ not $\backslash$ know $\backslash$ top

不知知病 not $\backslash$ know $\backslash$ know $\backslash$ sick

夫唯病病故不病 $-\backslash$ only $\backslash$ sick $\backslash$ sick $\backslash$ thus $\backslash$ not $\backslash$ sick

We can translate this as follows:

To know that you don't know is best.

(or: To know and to believe not to know is best.)

Not to know that you know is sick.

(or: Not to know and to believe to know is sick.)

Only who recognizes this sickness as such is not sick.

(or maybe: Who is only sick of this sickness is not really sick.)

Pondering a little about this example should make clear how much has been inserted in the translation. The other example, from Zhuangzi, is this one:

生生者不生 life \life $\backslash-\backslash$ not $\backslash$ life

which can be translated as:

What gives life to what is living does not live itself.

This example nicely shows how one "word", namely 生, can function in different ways: The first 生 can be read as a transitive verb meaning "to give life to". The second 生 can be read as a noun (object) meaning “the living", or "what is living". The third 生 can be read as an intransitive verb, meaning “to live”. (The particle 者 turns the whole 生生 into a noun: that which gives life to what is living. 生生者 then becomes the subject of the sentence.)

Now we can come back to the question of whether Chinese has subject and predicate form. In the above sentence, the first 生 functions as a transitive verb of the first part, 生生 (to give life to what is living). In combination with 者 this becomes a noun phrase and the subject of the sentence. 
The second 生 functions as noun and object (what is living) with respect to the first 生 (to give life to). The third 生 functions as intransitive verb and predicate of the whole sentence (to live). So, if we view 生 as a single word, out of context and in isolation, we cannot say whether it is a noun or a verb. But in the sentence above it takes on certain functions that we can well regard as subject or predicate. The function seems to be there, but not the class! Words do not nicely fall into classes. If you try to put them into such classes, they drift from one into another. At best, they have tendencies to preferably fulfill this or that function. The Chinese therefore have never classified their words into such classes.

Inflections would indicate classes, but we have seen that there are none in Chinese. One therefore might wonder how "real" the functions are. They come out if we translate, into English or French for example. But if we don't, then they simply do not "come up". Are they then "hidden”, under the surface, or are they not "real"? Certainly they are not as real as in languages where we have classes of words that go together with such functions, the classes of verbs and the classes of nouns. One can write down a list of verbs, but it is more difficult to write down functions. Functions are more abstract, and they need contexts to express themselves.

Harbsmeier brings a nice analogy here. He compares words in, say, Latin or Greek, with the figures in a chess game, and words in Chinese with the players in a soccer game. In chess, each figure can make only certain moves according to certain rules, whereas in soccer, a player, say from the defense, can, if the opportunity presents itself, run ahead, cross the field all the way, and score a goal. He usually has the function of defense, but he might change that for another in certain circumstances. The difference between class and function is fundamental to Chinese, so it seems to me, and maybe it has subtle consequences for the user of this language. We will come back to this.

Wilhelm von Humboldt, whose ideas I have been using a great deal up to now, in his long French letter to Abel-Rémusat of March 7, 1826, characterized the Chinese language as being “isolating”. It isolates words, concepts, and meanings. Given the monosyllabic character and the lack of cross-referential inflections of the Chinese language, this claim makes much sense to me. One has to move in and out of the language itself. One cannot "unfold" the meaning of a sentence by paying attention to the inflections, as one can in Latin. There one can do this, at least to some degree, even if one does not know the meanings of several words. One can go by 
the suffixes. One can let oneself be guided by the inflections (the systematic scheme of variation SSV). Chinese has syntax and particles, but no inflection. At this point one might wonder whether this implies that one needs to move farther out from the formal grammatical features and into the world of meanings and (social and textual) contexts. Of course this would only be a difference in degree. Consequently, proponents of an externalist theory of meaning might happily conclude that Chinese would be more "meaning-externalist” than Western languages.

We now can make the following tentative claim:

C1: Due to its being "isolating", Chinese might require the language user to be more context sensitive, both within the text as well as outside in the world where the language is used and where the "meanings" are.

We can add a second, tentative claim to this. Let us think again about the special semantic + phonetic (SP) structure of the Chinese script. A Chinese character refers to the meaning of the word partly through its phonetic part (P) and thus indirectly via the spoken word; and partly and more directly through its semantic part $(\mathrm{S})$. We thus have a triangle: Starting from the written word (A), which has the SP structure, we have two lines, one to the pronunciation $\mathrm{B}$ (guided by $\mathrm{P}$ ) and from there on to the meaning $\mathrm{C}$, and another directly to $\mathrm{C}$ (guided by $\mathrm{S}$ ).

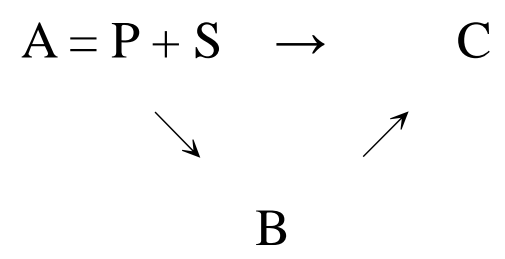

This triangular structure should play a role in the thinking of someone who writes Chinese. Although it is difficult to say just how much of a role it does play, the SP-structure of the script is certainly there: Lexicography organizes the words around the semantic parts, and people use dictionaries by looking up those semantic parts (the radicals), if they see a character they do not know.

In Western (alphabetic) languages, we have, as we pointed out in Aristotle above, a linear structure. The written word (A) stands for the spoken (B), which in turn stands for the meaning (conflating for a moment the soul 
and the world as Aristotle mentioned them). There is no direct line from A to $\mathrm{C}$. There is no semantic S-part in A:

$\mathrm{A} \rightarrow \mathrm{B} \rightarrow \mathrm{C}$

Looking at these diagrams, we see the difference between the triangular and the linear structures of reference, which leads me to the second tentative claim.

C2: Due to the semantic part in its script, the Chinese language involves the reader and writer of Chinese more directly in the world of meaning and objects.

I have taken the triangular structure between script, sound, and meaning from an article by Tze-Wan Kwan (2001). He has perhaps overestimated the significance of the semantic part, S. After all, S is only one part of a Chinese character, and the associated field of meaning is vague. Chinese characters are not pictures. Only parts of them (the semantic parts) are somewhat pictorial.

Comparing the two claims, we see that claim C2 is minor when compared with C1. It applies only to the script and to those who use it, whereas C1 applies to the language as a whole, not just in its written form but also in its spoken aspects. The feature of being isolating (monosyllabic, without inflections) is more fundamental, so it seems to me.

If there is less grammar, then the language projects less onto the world, that is, it has less of a systematic scheme of variation (SSV), and it is more open for the language user to induce the connections between its words from the outside, that is, from the world, on that occasion. To put it schematically, instead of

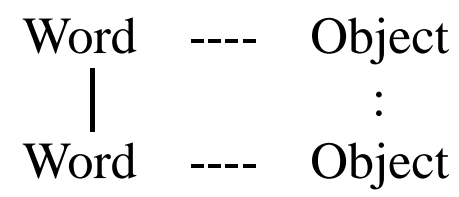

where the formal grammatical connection between the words is projected from the language into the world, we have: 


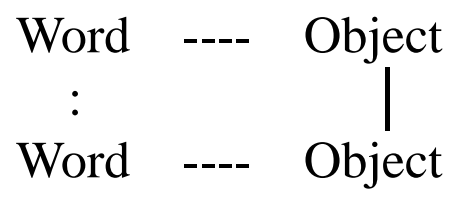

where the material, real connection between the objects in the world is induced into the language.

If a language tends to minimize the number of words it uses in a sentence, and classical Chinese very much does, and very elegantly so, then it puts a greater burden on the reader and listener to fill in what has to be understood from context and situation. This also applies to its active users, those who write or speak, because they have to put themselves into the position of the reader and listener if they want to make sure that they will be understood. This applies to any language, but it does so more prominently in Chinese, so it seems to me.

The fact that there are functions instead of classes of words has consequences. Chinese is more contextual, interpretive, open, flexible, and holistic than Western languages. It is based more on roles than on rules. I know that all of this is vague, but I think that what I have indicated about the structure of Chinese above does give some substance to this. We are talking here of tendencies, not strict determinations or impossibilities. Languages are elastic. Given enough space and time, one can do almost anything in one language that one can do in another. The problem is space and time. Unique tendencies exist within particular languages. Some languages are better in certain things than other languages. They allow one to do certain things more easily, more conveniently, and with less effort. They create affordances. Going hand in hand with this, they might also require certain things that other languages don't require. Requirements and affordances are tendencies, but they create habits of thought, which in turn affect the mind. In the next section, I wish to bring this out more clearly.

\section{Language affects the Mind? (Sapir-Whorf) Empirical Evidence}

Before drawing a picture in broader strokes, I would like to indicate an experiment (regarding two particular words) that gives some evidence to the Sapir-Whorf hypothesis. It suggests how language in certain ways "determines” thought. This leads us to think that something similar could be 
the case, not regarding single words, but regarding grammar, which is the point of my paper.

We often talk about temporal relations in terms of spatial metaphors. English and German use horizontal ones: We look forward to a better future. The worst is still ahead of us and not yet behind us. Back in the good old days things were different. Before you leave, please think for a moment. Similarly, in German we use the words: bevor, hintennach, hinterher, voriges Mal, zurückliegen. Chinese uses horizontal terms as well: 前 (in 前天, the day before yesterday) and 後 (in 後天, the day after tomorrow), but it also uses vertical ones: 上 (top, up, above) and 下 (bottom, down, below). So one says 上個星期 (“up week”, meaning last week), 下個星 期 (“down week”, meaning next week), and 上個月 (“up-month”, meaning last month) 下個月 (“down month”, meaning next month). Time does not only go by, it goes downwards, too.

Does this affect the way that speakers of Chinese think about temporal relations? Lera Boroditsky (2001) showed that native Mandarin speakers are influenced even when they are asked in English (when they "think for English”). They were shown vertical and horizontal arrays of objects and in between they were asked various questions, such as whether March comes earlier than April. They were faster in their response after having seen vertical arrays. For native English speakers the result was the opposite. Bilinguals fared in-between, according to the age at which they had begun to learn English. Then native English speakers were (somewhat artificially) taught to speak about time in vertical terms, and they then tended to behave like native Mandarin speakers in these tests.

Language creates habits and patterns of thought, and if we take stereotype- and prototype-theories of concepts seriously (according to which mental images play a central role in our having concepts of things), results of the kind pointed out above should not come as a surprise. Of course, the Sapir-Whorf hypothesis is still disputed and not settled. Nevertheless, a good number of experiments have recently come up with good evidence in favor of it. A good collection of reports of such experiments can be found in Gentner and Goldin-Meadow (2003). I do not want to go into further experiments here. It should be more or less clear that language plays a role in our thinking. The question is to what extent, and in what sense, we can say that it "determines" thinking, and what the deeper, or broader, consequences would be if that were the case. The point I am trying to make here is that there is something fundamental about the Chinese language that is 
different from what we find in Western languages. This fundamental difference consists not just in the use of words like 上 (up) and 下 (down) to express temporal relations. What I am after here are the implications of the grammatical (not lexical) differences between languages.

The grammatical structure and the isolating character of Chinese that I have pointed out above are not local. They are not bound to particular words that reflect particular conceptualizations. Rather, they are everywhere in Chinese. They are, so it seems to me, more at the root of a language. I do not know of any empirical study that tries to bring out the implications of these general grammatical differences for the way language users think. Nevertheless, it would be most interesting if such an experiment could be carried out along the lines of the Sapir-Whorf hypothesis.

In the sections above, we have seen some linguistic differences between Chinese and Western languages on the one hand, and some psychological world-view differences between Asians and Westerners on the other. I have done this in such a way that certain parallels suggest themselves: On the one hand, the Chinese language tends to require its user to be more context sensitive; on the other, there is evidence for Asians being more perceptually sensitive than Westerners to background and interrelations in their understanding of the world. The question now is whether there is a connection, an inner causal connection and not just an outer accidental parallel, between the two: between the requirements that the Chinese language imposes on its users, and the higher perceptual sensitivity of Asians. The Sapir-Whorf hypothesis would seem to suggest that there is such a connection.

But the differences in perceptual sensitivity that surfaced in the experiments pointed out above might have all kinds of cultural and educational reasons and causes. How can we know that language is one of them? Furthermore, I have only reported a handful of experiments, and these involve not only Chinese, but also Japanese and Koreans, and they do not speak Chinese. Although Japanese still uses some Chinese characters, it now also uses syllabic writing (Katakana and Hiragana) in its script. Korean formerly used Chinese characters, but now has a phonetic script (Han'gǔl). They do not have a Roman alphabet, yes, but they do have phonetically based scripts, whereas Chinese has a morpheme- or word-based script. They do not inflect, yes, but they agglutinate instead, whereas Chinese is an isolating language.

If Korean and Japanese are closer to Western languages and do not have 
the linguistic features I have pointed out for Chinese (monosyllabic, lack of inflection), then it might be more likely that the parallels (between Asian perception and the Chinese language) are merely accidental. The perceptual behavior of Koreans and Japanese might then be due to other factors, not to language, and in particular not to the Chinese language. Furthermore, if this is true, it might hold for the Chinese as well: They might perceptually behave in certain "Asian” ways (together with the Japanese and Koreans) due to reasons that have nothing or little to do with the language itself.

On the other hand, trying to give support to Sapir-Whorf again, Korean and Japanese ways of behaving and seeing the world, their culture, aesthetics, arts, values, social structures, education, etc., have been strongly influenced by the Chinese, and these in turn might indeed have been influenced by their language in the way I have suggested. Thus we would at least have an indirect, namely historical influence.

To go one step further, on the opposing side, someone might object that Chinese today is not the same as the classical Chinese of 2000 years ago, where the monosyllabic structure was more dominant, so that the features of the Chinese language I pointed out would be of little consequence today. This person might also add that many Chinese cannot read or are otherwise not sensitive to those features. To all this I would answer, firstly, that even modern Chinese is at its heart monosyllabic, and second, that Confucius certainly was sensitive to those features and that many Chinese have read Confucius since then and have set up systems of education under his influence. The traces of Confucius exist, more or less, everywhere in Chinese society, even today. The traces do not fade; they evolve. This also applies to Japan and Korea, maybe gradually also to the West, when Chinese views begin to enter Western societies. The Sapir-Whorf hypothesis thus shows a new face, a historical one.

Korea and Japan are influenced by the Chinese and by the Chinese language. Nevertheless, they do not speak Chinese. This casts an oblique light on the parallels between Asian perception and the Chinese language pointed out above. China is not all of Asia. Nor is Asia "the East". There is also India, and as Sanskrit has a rich morphology, we must be even more careful here and avoid over-generalizations and hasty conclusions.

Despite these complications, I still think it makes good sense to ask whether there are causal relations between the linguistic and the psychological. If there are, and if we look at language and culture in evolutionary perspectives, then those causal influences go both ways, from culture to 
language and vice versa. But if a language has already evolved and "decided”, as Humboldt sometimes likes to put it, to stick to and further develop certain features (in the case of Chinese: being monosyllabic, isolating, and having characters for its script) and not to develop others, then this can set up certain structures that are impossible to trace back and re-develop differently. It can be like a tree branching out. Branches do not naturally shrink back. Furthermore, once they begin to grow, they grow bigger and bigger and smaller branches begin to grow from them. Chinese grammar might be such a parting point.

If all this makes sense and if we keep in mind the growing economic and political strength of China versus the USA and Europe, then we have an interesting perspective. We will have a point in saying that there remains a difference (in tendency) between China and the West, at least as long as Chinese and Western languages, as we have them now, in particular their grammar, live on, side by side. Of course these languages will further develop and influence each other. But that is another story.

For helpful comments, I thank Elmar Holenstein, Joel Schickel, Sho Saito, Erhard Scholz, Lai Chiu-Yueh, and Robert Reynolds. For financial support I thank the National Science Council of Taiwan and the Austrian Wittgenstein Society.

\section{REFERENCES}

Aristotle On Interpretation, translated by H.P. Cooke, The Loeb Classical Library 325, Cambridge/Ma: Harvard University Press 1938.

Boroditsky, L. 2003 “Linguistic Relativity”, in L. Nadel (ed.), Encyclopedia of Cognitive Science, John Wiley \& Sons.

- 2001 "Does Language Shape Thought?: Mandarin and English Speakers' Conceptions of Time”, Cognitive Psychology, 43, 1-22.

Gentner, D. and Goldin-Meadow, S. (eds.) 2003 Language in Mind. Advances in the Study of Language and Thought, Camebridge/MA: MIT.

Harbsmeier, C., see Humboldt.

Humboldt, Wilhelm von. Brief an M. Abel-Rémusat über die Nature grammatischer Formen im allgemeinen und über den Geist der chinesischen Sprache im besonderen. Nach der Ausgabe Paris 1827 ins Deutsche übertragen und mit einer 
Einleitung versehen von Christopher Harbsmeier / Christopher Harbsmeier. Zur Philosophischen Grammatik des Altchinesischen im Anschluß an Humboldts Brief an Abel-Rémusat. Grammatica Universalis 17. Friedrich Fromman Verlag, 1979.

Kwan, T.-W. 2001 "Wilhelm von Humboldt on the Chinese Language - Interpretation and Reconstruction”, Journal of Chinese Linguistics, 29, no. 2, 169-242.

Nisbett, R.E. 2003 The Geography of Thought: How Asians and Westerners Think Differently ... and Why, New York: Free Press.

Sampson, G. 1985 Writing Systems. A Linguistic Introduction, Stanford: Stanford University Press.

Wittgenstein, L. 1958 Philosophical Investigations, translated by G.E.M. Anscombe. Second edition, Oxford: Blackwell. 